\title{
Geometric part of uncertainties in the calculation constant of the primary four electrode conductivity cell
}

\author{
Alexander Mikhal $^{1}$, Zygmunt Warsza ${ }^{2}$ \\ ${ }^{1}$ Institute of electrodynamics National Academy of Science, Av. Peremogy 56, 03680 Kiev, Ukraine \\ ${ }^{2}$ Industrial Research Institute of Automation and Measurements PIAP, Al. Jerozolimskie 202, 02-486 Warszawa Poland
}

\begin{abstract}
The paper presents the construction of a primary four electrode conductivity cell with calculated constant for the Ukrainian primary standard of electrolytic conductivity (EC). The equations for calculating the cell constant and the error budget for calculating uncertainty are presented. The components of the budget are: errors due to the non-uniformity of the force lines of the electric field; errors due to the accuracy of measurement standards and measuring instruments for determining length and diameter of the tube; and errors due to manufacturing techniques of tubes and their assemblage. The article considers in detail the errors due to the non-ideal profile of the central part of the tube. Two methods to reduce the standard deviation are given: the method of linear interpolation for compensation of the concave form which occurs along the axis of the tube and the method of equivalent triangles to compensate for deviations from a circle that occur across the axis of the tube.
\end{abstract}

Section: RESEARCH PAPER

Keywords: conductivity; primary cell; geometric errors; standard deviation

Citation: Alexander Mikhal, Zygmunt WARSZA, Geometric part of uncertainties in the calculation constant of the primary four electrode conductivity cell, Acta IMEKO, Acta IMEKO, vol. 4, no. 2, article 4, June 2015, identifier: IMEKO-ACTA-04 (2015)-02-04

Editor: Paolo Carbone, University of Perugia, Italy

Received April 30, 2014; In final form July 13, 2014; Published June 2015

Copyright: @ 2015 IMEKO. This is an open-access article distributed under the terms of the Creative Commons Attribution 3.0 License, which permits unrestricted use, distribution, and reproduction in any medium, provided the original author and source are credited

Funding: This work was supported by the Presidium of the National Academy of Sciences of Ukraine.

Corresponding author: Alexander Mikhal, e-mail: a_mikhal@ukr.net

\section{INTRODUCTION}

In the recent years, the leading economically developed countries have established national standards of electrolytic conductivity (EC), the basis of which uses the "absolute (direct)" method of reproduction of a physical quantity [1]. World practice knows a lot of ways to implement this method; however, the principle of its operation is almost the same [2-4]. The "absolute (direct)" method is based on the measurement of the liquid column resistance and on the calculation of the EC by the known length of the column cross-sectional area.

The basic components of the EC standard of Ukraine are a four-electrode cell with the calculated constant, a special conductivity AC bridge, a thermostat for temperature control at $25^{\circ} \mathrm{C}$ and a precision digital temperature meter. The EC is determined in accordance with the expression:

$k=g K\left(1+\alpha \Delta t_{25}\right)$

where $g$ is the conductivity of the liquid column obtained as a measurement result of the conductivity bridge;
$K$ is the cell constant obtained as a calculation result based on the actual geometric dimensions of the cell;

$\alpha$ is the temperature coefficient for conductivity of potassium chloride solution;

$\Delta t_{25}$ is the temperature deviation from $25^{\circ} \mathrm{C}$ obtained as a measurement result using the digital thermometer.

The uncertainty (here referred to as standard uncertainty type B only) of the national standard will be influenced by many factors. Most significant errors are the ones in calculating the primary cell constant. Error components being determined by the geometric parameters of the liquid column have the greatest influence. Let us consider these error components on the example of the four-electrode conductivity cell which is used in the EC standard of Ukraine [4]-[6]. The basis of the conductivity cell is the sensor element, a sketch of which is shown in Figure 1.

The sensor element is a tube with internal diameter $D$. The inside of the tube is filled with an electrolyte solution. Typically, this is a solution of potassium chloride. The tube is used to fix the geometry of the liquid conductor and consists of three parts. The central part of the tube 1 has length $L$, two side 


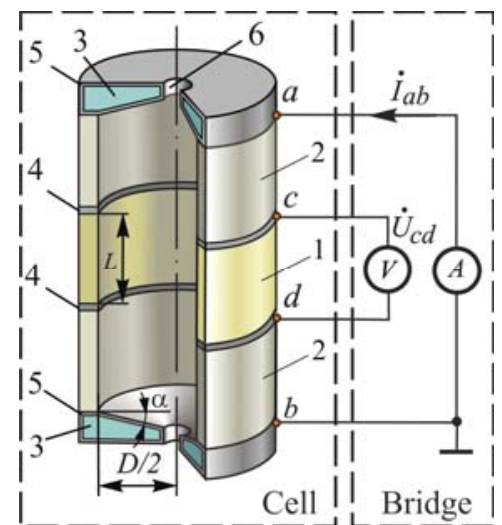

Figure 1 . Construction of the primary conductivity cell.

portions 2 have equal lengths $l$. The ends of the central portion of tube 1 are coated with circular potential electrodes 4 . Their width corresponds to the tube wall thickness. Two discs 3 are fixed at the edges of the tube. The inner surface of the discs is coated with a metallic film 5 which performs the function of current electrodes. Discs 3 have central holes 6 with diameter $d$ which serve for filling with the liquid. The inner disc surface has the form of a cone with angle $\alpha$. Such configuration is intended to facilitate the removal of air bubbles when filling the cell with the liquid. The tube and the discs are made of quartz glass which has good insulating properties, temporal stability and a minimum coefficient of thermal expansion. Platinum is used as the metal for the electrodes as it has a minimum polarization effect for most electrolytes. The cell in its four points $a, b, c$ and $d$ is connected to the bridge which ensures the measurement of conductivity of the liquid column.

The cell constant is determined by calculating the ratio of the length of the tube 1 to the cross-section area. However, this definition is true for an idealized object of measurements with uniform distribution of current flow lines. Distortion of such lines will be due to: the presence of holes for solution filling; the form of current electrodes and the presence of potential electrodes; and the non-ideal profile of the inner quartz tube 1, Figure 1. Therefore, the calculation of the constant will show errors.

\section{BUDGET OF UNCERTAINTY CELLS}

The error budget for the constant calculation of the cell, shown in Figure 1, can be written as [7]:

$u_{K}=F\left[\delta_{S t}, \delta_{\text {Cal }}, \delta_{\text {Geom }}\left(\delta_{\text {Tec }}, \delta_{P E}\right)\right]$

where: $\delta_{S t}$ is an error due to the accuracy of measurement standards and measuring instruments that determine the length and diameter of the tube;

$\delta_{C a l}$ is an error due to the deviation of the calculation model for the cell constant in real conditions relative to the idealized model;

$\delta_{\text {Geom }}$ is an error in assessment of geometrical dimensions.

We do not focus our attention on the method of processing errors (function $F$ ), which is used in calculating the uncertainty. This is regulated by international guidelines [8].

Each error in Eq. (2) has in its turn a number of components.

Minimizing of the error $\delta_{S t}$ is limited by the level of metrological assurance for measurements of tube length and diameter. It is defined by the metrological characteristics of the standards and instruments for length measurement.

Error $\delta_{C a l}$ has two origins and two components accordingly: an error due to the alternating current measurement and an error due to the discontinuity of an electric field in the cell because of its finite dimensions and design features.

The geometric error $\delta_{\text {Geom }}$ also has two components:

$\delta_{T e c}$ is an error due to manufacturing technology for the tube sections and their assemblage;

$\delta_{P E}$ is an error due to the presence of the potential electrodes.

The latter component of the error depends on the finite thickness of the potential electrodes and on changing position of a singular point of the potential electrodes upon assemblage. This component is related to the calculation of the electric field inside the cell. It will be considered in other papers. In this paper, we examine the component of error $\delta_{T e c}$. This error is due to the deviation of the actual profile of the inner surface of tube 1 (Figure 1) from the ideal profile of the tube. The latter one is presented as a rectangle along the longitudinal section and as a circle in cross-section.

It should be mentioned that the cost of tube production from a monolithic quartz crystal is extremely high. As a rule, tubes are manufactured from work pieces (preform) which undergo precision machining. If precision machining of the inner surface is too deep, the mechanical resistance of the tube will reduce significantly. Tubes of less than $1 \mathrm{~mm}$ in thickness will crack (fracture) under elastic forces (adhesive polymerization, temperature differences). Therefore, grinding of the work piece inner profile should be of minimum depth. On the other side, the work piece inner surface can have wedge-like cracks which are in parallel to the axis of the work piece. These cracks are due to manufacturing techniques of the work piece production and depend on the quality of nozzles through which the work piece is pulled. Therefore, due to the lack of deep machining of tubes, we can observe deviations from a circle in cross-section along the entire profile. The second reason of a non-ideal profile may be the precession of the grinding tool. During processing, quality control of the tube is practically impossible. After final grinding, the tube profile may differ from the ideal rectangle.

\section{ERROR DUE TO MANUFACTURING}

To determine the actual profile of the tube, its diameter and length should be measured according to the following algorithm. We performed $p$ measurements of the tube length $L$ in different directions. These directions were distributed uniformly around the circumference. Conventionally, uniformly along the length, the tube is divided into $m$ sections. To define the diameter, $n$ measurements are made in the cross-section of each part of the tube in different directions. As a result, we obtain $n \times m$ measurements of the tube diameter and $p$ values of the tube length. The constant can be determined from the measurement results through averageing the values of diameter $D_{a v}$ and tube length $L_{a v}$.

$K=\frac{4 L_{a v}}{\pi D_{a v}^{2}}$

In modern technologies for processing quartz glass it is much easier to manufacture a tube with stable length value than a tube with stable internal diameter. From the experimental data we observe distortions of the internal profile of two types. The 
first one is a deviation of the tube profile from a rectangle along the longitudinal section due to precession of the grinding tool. The second one is a deviation of the tube profile from a circle in cross-section due to the presence of wedge-like cracks on the inner surface of work pieces.

The geometric dimensions of the actually manufactured tube can be measured with much higher accuracy than the error of profile. Parameter measurements are performed with a device with an LSB of $0.1 \mu \mathrm{m}$. Random components of the device are negligible. The actual tube profile deviates from the ideal rectangle by more than $10 \mathrm{LSB}$ of this device. Therefore, the following expression can be taken as a metrological model for the constant calculation:

$$
K=K_{0}\left(1+\delta_{K}\right), \sigma_{K}=\sqrt{2 \sigma_{D}^{2}+\sigma_{L}^{2}}
$$

where: $K_{0}$ is the true value of the constant which is determined by the actual profile of the tube inner surface;

$\delta_{\mathrm{K}}$ is a systematic relative error of the constant calculation;

$\sigma_{K}, \sigma_{D}$ and $\sigma_{L}$ are standard deviations of the mean cell constant, diameter and length of the tube, respectively.

Therefore, an error due to manufacturing $\delta_{T e c}$ has two components: a systematic $\delta_{\mathrm{K}}$ and a random $\sigma_{\mathrm{K}}$.

Hereinafter, under such deviation we understand the standard deviation of the mean (SDM) of the diameter. The average diameter and the SDM of the diameter in each $i$-th section of the tube are determined from the expressions:

$$
\begin{gathered}
D_{a v}=\frac{\sum_{i=1}^{m} D_{i, a v}}{m}=\frac{\sum_{i=1}^{m} \sum_{j=1}^{n} D_{i j}}{m \cdot n} \\
\sigma_{i D}=\sqrt{\frac{1}{n(n-1)} \sum_{j=1}^{n}\left(D_{i j}-D_{i a v}\right)^{2}}
\end{gathered}
$$

where: $D_{i, a v}$ is the mean diameter in the $i$-th section of the tube and $D_{i j}$ is the diameter in the $j$-th direction and in the $i$-th section of the tube. The SDM value from the $m$ section (cut) can be expressed as:

$$
\sigma_{D}=\sqrt{\frac{1}{m(m-1)} \sum_{i=1}^{m} \sigma_{i D}^{2}}
$$

\subsection{Error in the longitudinal section}

The measuring results for the mean diameter of one of the sections of the tubes for $n=8$ and $m=10$ are shown in Figure 2.

These data indicate that the average diameters in sections 1 and 6 differ by almost $20 \mu \mathrm{m}$. In general, the profile of the tube internal section can be expressed through an arbitrary function $D(x)$. The measuring results for average diameters along the length of the tube (Figure 2) show that this dependence has



Figure 3. Profile along the axis of the tube.

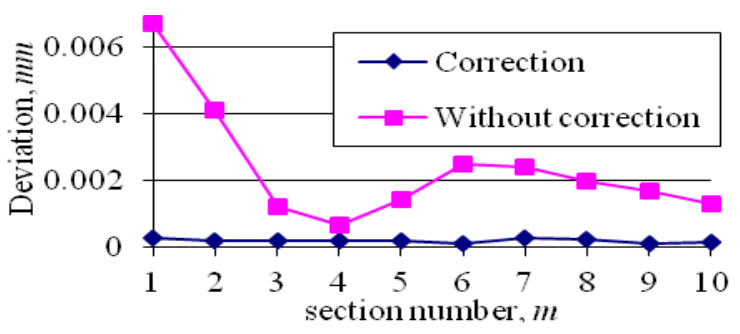

Figure 2. Influence of linear interpolation on the level of SDM $\left(\sigma_{i D}\right)$.

clearly a deterministic character. The discrete nature of the data allows us to use linear interpolation for the function $D(x)$. The results are shown in the following formula:

$K=\frac{4}{\pi} \int_{0}^{L} \frac{d x}{D(x)^{2}}=\frac{4}{\pi} \sum_{i=0}^{m} \int_{0}^{\Delta X_{i}} \frac{d x}{(a x+b)^{2}}$

where: $a$ and $b$ are linear interpolation coefficients;

$\Delta X_{i}$ the length of the region between the $i$-th and $i+1$ section.

The polynomial coefficients are expressed as:

$a=\frac{D_{i+1}-D_{i}}{\Delta X_{i}}$

$b=D_{i}$

After simple transformations, the expression for calculating the constant with the proposed correction takes the form:

$K=\frac{4}{\pi} \sum_{i=1}^{m} \frac{\Delta X_{i}}{D_{i, a v} D_{i+1, a v}}$

We can use equation 3 and 5 , but then we get an increase in the standard deviation. Let us show in one figure the graphs for the SDM of diameter measuring results with and without the deterministic component by using linear interpolation (Figure 3). As it can be seen, the SDM of the diameter measurement results, taking into account the deterministic component, is close to almost one value and has a very small scatter of results compared with the case where the deterministic component is ignored and the average diameter value is calculated according to Eq. (3). As a result of this correction, the change of the SDM along the cross-section is reduced by 10 to 15 times. The SDM of the diameter with correction, calculated according to Eq. (5c), is less than $0.0004 \mathrm{~mm}$. The same parameter without correction is 3 times larger. When calculating the random component of an error (Eq. 4), we obtain an error reduction by two.

It should be noted that such correction method shifts the average value of the diameter. Thus, the constants calculated by Eqs. (3) and (7) for tube 1 in Figure 1 differ by $0.027 \%$. This is the rate by which systematic relative errors $\delta_{\mathrm{K}}$ differ (4) when calculating the cell constant with and without correction.

\subsection{Error in the cross-section}

Let us consider another tube for which the values of the mean radius in each of the sections are grouped along a virtually horizontal line. However, in each individual section the surface profile differs from a circle. Example of the function for the deviation from the mean $D_{\text {iav }}$ (figure represented by circle $\left.0.5 D_{3, a v}=4.569 \mathrm{~mm}\right)$ in one of the sections with $m=3$ is shown in Figure 4.

In all ten sections with $m=1$ to 10 we observe triangular run outs along the lines 3 to 11 and 6 to 14 (Figure 4). Such 


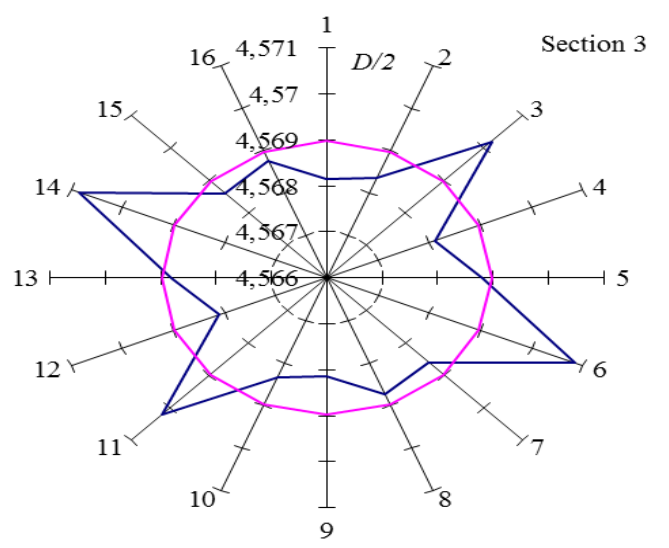

Figure 4. Real profile (blue) across the axis of the tube for section $m=3$ and $0.5 D_{i, a v}=4.569 \mathrm{~mm}$ (red).

character of profile distortions allows using a method of the equivalent triangles. This method involves the assessment of the effective area $S_{i e f}$ of the tube section and subsequent diameter corrections. The algorithm for calculation is to replace the diameter values in section 3 and 6 with the values of the mean diameter $D_{a v}$, then to use standard formula for calculating the basic mean diameter $D_{\text {bias }}$ and the basic area of the tube section $S_{\text {bias: }}$ :

$S_{\text {bias }}=\pi\left(D_{\text {bias }}\right)^{2} / 4$

Next, we calculate the correction value in each section separately. It is represented as areas of triangles:

$S_{i}=c_{i} h_{i} / 2$

$c_{i}$ is the base of the assumed triangle in direction 3,11 or 6,14 (Figure 4); $b_{i}$ is the height of the assumed triangle in the direction 3,11 or 6,14 (Figure 4).

We take into account the influence of the deterministic component by forming the effective area of each section which is expressed as:

$S_{i e f}=S_{\text {bias }}+2 \sum_{i=3,6} S_{i}=S_{\text {bias }}+\sum_{i=3,6} c_{i} h_{i}$

According to Eq. (11) we calculate the corrected $D_{\text {icor }}$ which is put in Eqs. $(2,3)$ instead of $D_{i a v}$ :

$D_{\text {icor }}=\sqrt{4 S_{\text {ief }} / \pi}$

The difference between SDM values for the measurement result of diameter $D_{\text {iav }}$ before correction and SDM values for the mean diameter $D_{i c o r}$ after correction is shown in Figure 5:

As can be seen in this figure, the SDM with the correction of the deterministic component is 2.5 to 3 times less than without correction. The use of an algorithm of effective areas shifts the mean diameter value. The constants calculated by

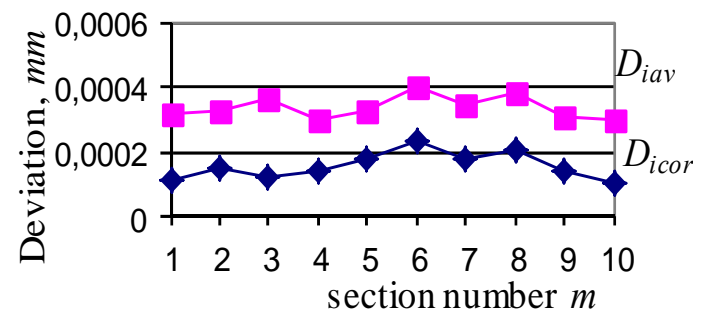

Figure 5. SDM $\left(\sigma_{i D}\right)$ without correction $D_{i a v}$ and with correction $D_{i c o r}$.

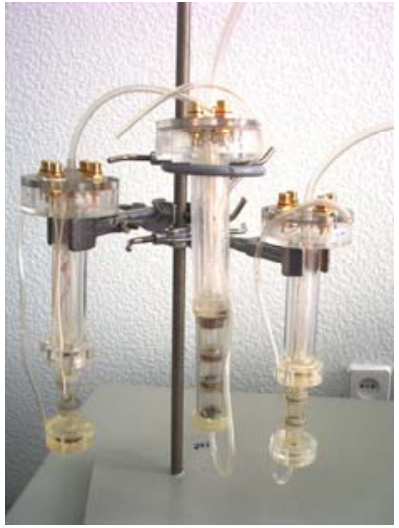

Figure 6 . The cells for the primary standard.

Eqs. (5) and (11) differ by $0.015 \%$. Just as in the previous case, this value represents the difference of systematic errors $(3,4)$ for the cell constant calculation.

\section{CONCLUSIONS}

The above methods were used to calculate the corrections to the cell constant. For the primary EC standard of Ukraine, several cell designs were made. The primary cell is shown in Figure 6. Correctness, sufficiency and adequacy of the all selected models for correction constant cells is confirmed by international comparisons P22, P47, K36, which involved primary standard of Ukraine (laboratory UkrCSM).

14 laboratories of the leading NMI of the world participated in international comparisons CCQM-K36: USA (NIST), Germany (PTB), Israel (INPL), Slovakia (SMU), Denmark (DFM), and others. To all participants samples of potassium chloride solutions with a nominal electrolytic conductivity 0.5 $\mathrm{S} / \mathrm{m}$ and $5 \mathrm{mS} / \mathrm{m}$ were sent. Each laboratory using equation (1) and own metrological features establishes the exact value EC $k_{l a b}$ and uncertainty $u\left(k_{\text {lab }}\right)$ conductivity samples.

The rules of Key Comparison specify that a Key Comparison Reference Value must be derived against which the participants' results are compared, and a Degree of Equivalence of each laboratory must be inferred. In [6] the model of Reference Value $k_{\text {ref }}$ was presented. The Degree of Equivalence is given as:



Figure 7. The results of international comparisons CCQM-K36.a [6]. 


$$
D(S m / m)=k_{\text {lab }}-k_{\text {ref }}
$$

The results of the Degree of Equivalence with uncertainty on the international comparisons CCQM-K36 are shown in Figure 7. The proposed methods of correction constants cells (method of linear interpolation and method of effective areas) allowed us to solve two problems [7]. Firstly, the value obtained by the UkrCSM $k_{\text {labs }}$, practically coincides with the value $k_{r e f}[6]$. Secondly, by minimizing the random component of the error (Eq. $4,5 \mathrm{c})$, a minimum value of uncertainty $\mathrm{u}\left(k_{\text {lab }}\right)$ was obtained.

\section{ACKNOWLEDGEMENT}

The authors express their gratitude to prof. M.N. Surdu, as supervisor of researches [4, 5] and V.G. Gavrilkin, as a scientist keeper of primary standards [5, 6], Head of the Department of metrological provision of physical and chemical quantitative measurement (lab. UkrCSM, Kiev).

\section{REFERENCES}

[1] Wu Y.C., A DC method for the absolute determination of conductivities of the primary standard $\mathrm{KCl}$ solution from $0^{\circ} \mathrm{C}$ to $50^{\circ} \mathrm{C}$, J. Res. Nati. Inst. Stand. Tecnol., 1994, 99, №3, 241-246.
[2] Marriarssy M., Pratt K.W., Spitzer P., Major applications of electrochemical techniques at national metrology institutes, Metrologia 46 (2009) 199-213

[3] Shreiner R.H., Pratt K.W., Standard Reference Materials: Primary Standards and Standard Reference Materials for Electrolytic Conductivity, NIST Special Publication 260-142, 2004 Ed.

[4] Brinkmann F. and etc., General paper: Primary methods for the measurement of electrolytic conductivity, Accred Qual Assur. 2003. - 8:346 - 353 DOI 10.1007/s00769-003-0645-5.

[5] Gavrilkin V.G. and etc., State primary standard unit of electrical conductivity of liquids, Ukrainian Metrology Journal, 2006, №3, P. 47-51. (Ukr.)

[6] Jensen H D 2006 Final Report of Key Comparison CCQM-K36. 15 August 2006, available online at http://kcdb.bipm.org/AppendixB/appbresults/ccqmk36/ccqm-k36_final_report.pdf

[7] Mikhal A.A., Warsza Z.L. Influence of geometric Uncertainties on the Accuracy of calculated constant of the primary conductivity cell. 11th International Symposium on Measurement and Quality Control. IMEKO, Poland, Cracow-Kielce, 11-13 September 2013, p.8

[8] Guide to the Expression of Uncertainty in Measurement, ISBN 92-67-10188-9, 1st Ed., International Organization for Standardization, Geneva, Switzerland, 1993. 